\title{
Sinonasal malignant melanoma: an analysis of 115 cases assessing outcomes of surgery, postoperative radiotherapy and endoscopic resection*
}

\author{
Valerie J. Lund ${ }^{1,2}$, Edward J. Chisholm², David J. Howard', William I. Wei ${ }^{3}$ \\ University College London, Ear Institute, London, United Kingdom \\ Royal National Throat, Nose and Ear Hospital, London, United Kingdom \\ Department of Surgery, Queen Mary Hospital, University of Hong Kong Medical Centre, Hong Kong SAR, China
}

Rhinology 50: 203-210, 2012

DOI:10.4193/Rhino.11.267

*Received for publication:

December 30, 2011

Accepted: February 10, 2012

\section{Summary}

Background: Melanomas account for $4 \%$ of sinonasal malignancies. We present the largest single institution series reported thus far and analyze the outcome with reference to lymph node involvement, radiotherapy and endoscopic resection.

Methodology: Survival and recurrence data were analyzed on sinonasal melanoma cases collected from 1963-2010 to compare treatment strategies and to ascertain factors predicting outcome.

Results: 115 cases (mean age 65.9) were treated at our institution during this period. All underwent surgical resection of the tumour, 31 (27\%) endoscopically, and 51 (44\%) also received radiotherapy. Five year overall survival was $28 \%$ and disease-free survival was $23.7 \%$. Local control was achieved for a median of 21 months, 5 -year disease control rate of $27.7 \%$. Endoscopically resected cases showed a significant overall survival advantage up to 5 years. Radiotherapy did not improve local control or survival. Cervical metastases conferred a dramatically worse outcome.

Conclusions: Endoscopic resection of sinonasal melanoma does not prejudice outcome. The role of radiotherapy is unproven.

Key words: sinonasal, nasal, sinus, melanoma, endoscopic

\section{Introduction}

Malignant melanomas are aggressive tumours arising from melanin-producing cells (melanocytes), derived from neural crest tissue. Cutaneous melanomas are the most common type of which $15 \%$ to $33 \%$ occur in the skin of the head and neck region ${ }^{(1)}$. Melanocytes are also present in mucosa, secretory glands, nasal stroma and supporting cells of the olfactory epithelium. They can undergo malignant transformation at any of these sites. These mucosal melanomas are rare accounting for $1.3 \%$ of all malignant melanomas; $55 \%$ are in the head and neck region ${ }^{(2)}$. Of these, two thirds originate in the sinonasal region and a quarter in the oral cavity ${ }^{(1,3-7)}$. Overall less than $1 \%$ of malignant melanoma are sinonasal ${ }^{(1,4,8)}$. Of the diverse range of sinonasal malignancies, melanoma accounts for approximately $4 \%{ }^{(9)}$.
Sinonasal melanomas are equally common in men and women. In previously reported series the mean age ( 64.3 years) has been older than those with cutaneous melanomas ${ }^{(1)}$. The incidence is much higher in Japan where mucosal melanomas make up a quarter to a third of all melanomas and they may be more common in black populations ${ }^{(4,10)}$.

The aetiology is unclear but pre-existing melanosis and formaldehyde exposure may be risk factors for developing sinonasal melanomas ${ }^{(1,11)}$.

The most common subsite for sinonasal malignant melanomas is the lateral nasal wall followed by, in order of frequency, septum, maxillary sinus and ethmoids ${ }^{(11)}$. They rarely originate in the sphenoid sinus, nasopharynx or nasal vestibule.

Presentation, as with other sinonasal malignancies, may mimic 
inflammatory conditions, leading to a delay in diagnosis. Unilateral nasal obstruction, epistaxis, a visible mass and pain are the most common features. The lesion may appear pigmented but frequently it is not.

Prognosis from sinonasal melanomas is poor, worse than its cutaneous counterpart. Five-year survival is typically less than $25 \%$, with reports varying between $8 \%$ and $48 \%{ }^{(1,6,9,11-20)}$. The cause of death is a consequence of both local recurrence and metastatic disease.

The mainstay of treatment for sinonasal malignant melanomas has been surgical resection, traditionally by an open approach but with a growing trend towards endoscopic techniques.

Numerous case series have looked at the addition of radiotherapy to improve outcome. There is a trend towards improved locoregional control with post-operative radiotherapy but no evidence to show prolonged survival ${ }^{(11,21,22)}$.

This study is an update 15 years after an earlier published series from our institution ${ }^{(17)}$ following a change in management strategy. Given the generally poor outcome for this tumour despite major open resections, from the mid-1990's it was decided to undertake an endoscopic resection irrespective of the extent of disease. The aim of this study is to ascertain if endoscopic surgery carries a worse prognosis when compared to the previous traditional open approaches. It also assessed the impact of postoperative radiotherapy and if there are any other factors in our data set that influenced outcome.

\section{Materials and methods}

Data collection

Data had been collected prospectively for 47 years (1963 to 2010) on all primary sinonasal melanomas treated at The Royal National Throat, Nose and Ear Hospital, London. Age, sex, primary site (when assessable), surgical management, post-operative treatment, recurrence and survival data were recorded.

\section{Surgery}

All cases included in the analysis underwent surgery with curative intent. A wide field mucosal resection was undertaken, mindful of the capacity of mucosal melanoma to have amelanotic areas and satellite lesions. This included open surgical approaches (lateral rhinotomy, midfacial degloving, maxillectomy and craniofacial resection) prior to 1996, which were undertaken by 3 senior surgeons (DFNH ${ }^{\S}, \mathrm{DJH}$ and VJL), and endoscopic resection after 1996 (with one exception) by one surgeon (VJL) irrespective of extent. This involved a similar extent of resection as that undertaken previously by external approaches, including medial maxillectomy, complete ethmoidectomy, anterior sphenoidectomy and wide marsupialisation of the frontal sinuses as dictated by the disease. However, orbital clearance was not undertaken in any endoscopic cases. Those with orbital involvement underwent resection of orbital periosteum and adjacent orbital fat, which achieved macroscopic clearance.
Similarly in those cases where skull base was eroded, dura with adherent tumour was resected and repaired primarily. None of the cases managed endoscopically had skin involvement. Selected cases received post-operative radiotherapy and / or chemotherapy.

\section{Statistical analysis}

As a consequence of the initial analysis in $1999^{(17)}$, which showed no statistical advantage for the addition of radiotherapy but indicated a small trend in its favour, the option of post-operative radiotherapy was discussed via a multi-disciplinary team with all subsequent patients, who could choose to have this or not. Consequently there was no blinding or randomisation of treatment, nor control group. Data was analysed using SPSS version 18.0 (IBM, Chicago, IL, USA). Outcome measures were local control (the endpoint being recurrence, specifically alive with disease or died of disease), overall survival (died of disease or another cause) and disease free survival (alive without disease). These were evaluated and compared by Kaplan-Meier curves and statistical significance was estimated by the MantelCox proportional hazard test. Comparison was made between lymph node positive and negative patients, post-operative radiotherapy and no post-operative radiotherapy and endoscopic versus open resection. Cox regression analysis was performed to identify other factors affecting outcome.

Since the early 1980 's all patients have been submitted to longterm follow-up. This includes regular outpatient attendance with endoscopic examination of the sinonasal cavity and imaging (MRI) every 3 - 4 months for the first 2 years, followed by 6 monthly up to five years and 9 - 12 monthly thereafter. Further endoscopic resection has been undertaken in patients who have developed local recurrence where appropriate, irrespective of the original surgical approach.

\section{Results}

One hundred and fifteen cases were analysed of which 64 were female (55.7\%) and 51 male (44.3\%). The mean age at the time of initial treatment was 65.9 years (range $15-91$ years). Ninety (78.3\%) cases originated in the nasal cavity, $12(10.5 \%)$ in the ethmoids with/without nasal cavity involvement and 7 (6.1\%) involved the maxilla (in 6 cases disease was too extensive to determine site of origin). At the time of diagnosis 101 patients (91\%) had no identifiable lymph node involvement (N0), 10 (9\%) had involved lymph nodes $(\mathrm{N}+)$ and the status of 4 of the early cases was unknown.

Follow up ranged from 2 - 360 months, mean 37.5 months in the 109 patients whose follow up was recorded. (Six patients were lost to follow up and were excluded from data analysis). The primary management in all cases was surgery. Lateral rhinotomy $(n=71)$ was undertaken in the majority of early cases, 4 had craniofacial resection, 7 radical maxillectomy and 


\section{Survival Function}

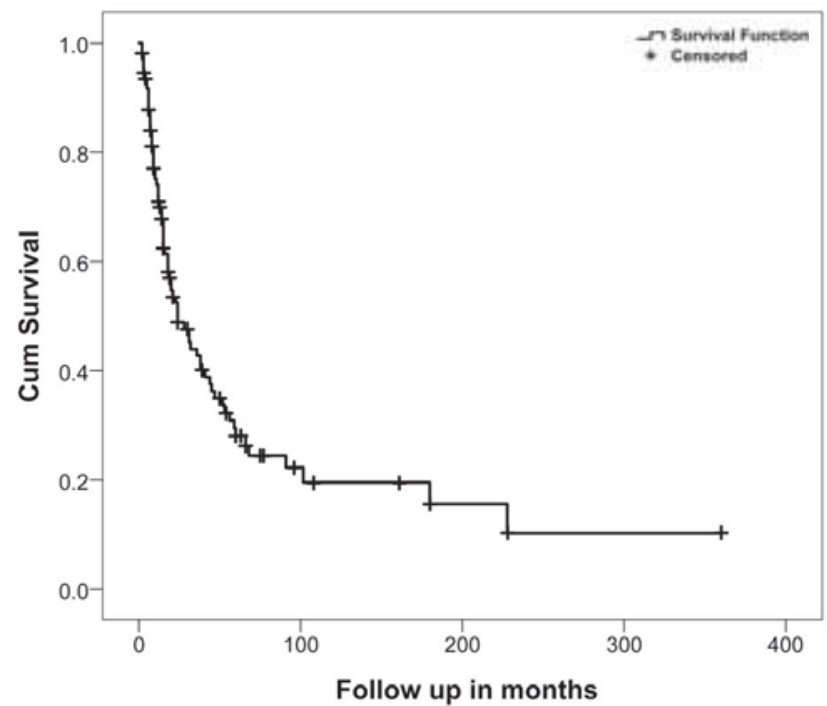

Figure 1. Kaplan - Meier graph of overall survival of 109 sinonasal malignant melanoma cases.

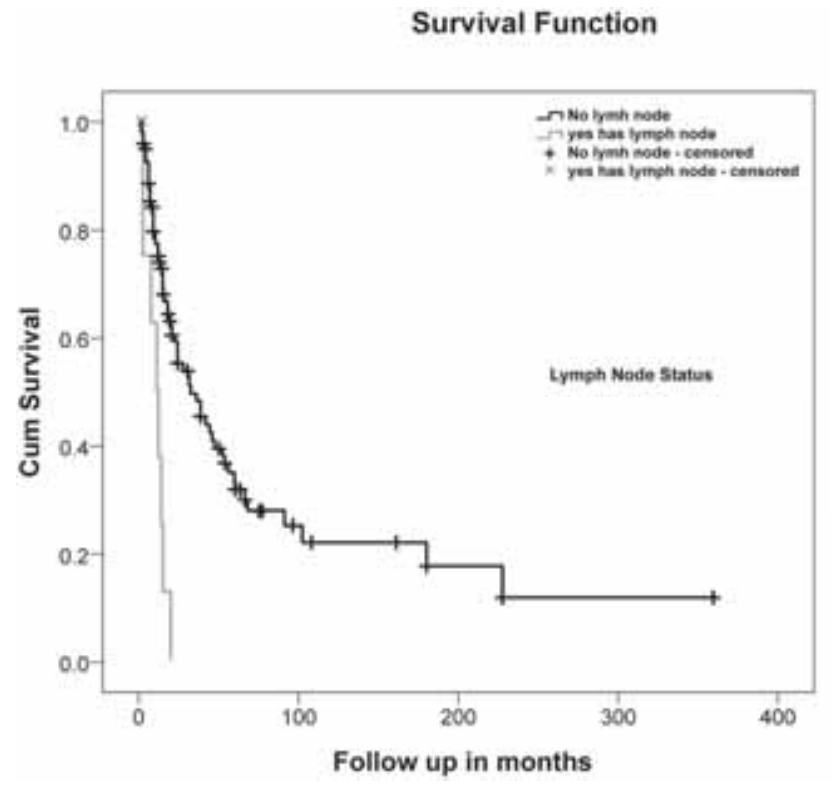

Figure 2. Kaplan - Meier graph of overall survival comparing cases of sinonasal melanoma with lymph node involvement $(\mathrm{N}+)$ at diagnosis to those without $(\mathrm{N}-)$.
4 underwent a midfacial degloving approach. These open techniques have been superseded by endoscopic resection (ESS) ( $n$ $=31$ ) in the latter series. The majority of the open procedures (78\%) and all of the endoscopic resections were undertaken by one surgeon (VJL).

Of the 109 cases with follow up data, 64 (55.7\%) had surgery alone and 51 (44.3\%) had additional radiotherapy (35 [45\%] open surgery; 16 [51\%] endoscopic surgery). Ten received chemotherapy in addition to the adjuvant radiotherapy (melphalan or cis-platin regimes) and 5 were given adjuvant chemotherapy alone ( 2 being Bacillus Calmette-Guérin, BCG) in the early 1970's. Since 1996, further endoscopic surgery has been undertaken in 12 patients, 9 originally treated endoscopically and 3 who had undergone previous lateral rhinotomy or maxillectomy, in some cases multiple times.

The patients were allocated into the following groups: alive without disease $(n=17,14.8 \%)$, alive with recurrence $(n=15,13 \%)$, died of intercurrent disease $(n=7,6.1 \%)$ or died of disease $(n=$ $66,57.4 \%)$. Ten further cases (8.7\%) were lost during follow up and were treated as 'censored' at the point of last follow up (as per Kaplan - Meier statistics, SPSS).

The overall median survival was 24 months (standard error (SE) $=5.127 ; 95 \%$ confidence interval $(\mathrm{Cl})=13.952$ - 34.048) (Figure 1). The 5 -year overall survival was $28 \%$ and 10 year survival $17.5 \%$. Local control was achieved for a median of 21 months
$(\mathrm{SE}=2.914 ; 95 \% \mathrm{Cl}=15.289$ - 26.711) with a 5-year disease control rate of $27.7 \%$. Median disease free survival was 21 months ( $\mathrm{SE} 2.943 ; 95 \% \mathrm{Cl}=15.232$ - 26.768) with a 5-year disease free survival of $23.7 \%$ and 10 years of $9.7 \%$.

If lymph nodes were involved at diagnosis $(\mathrm{N}+)$ the overall survival, local control rate and disease free survival were adversely affected (Mantel-Cox $\mathrm{p}<0.001)$. Median overall survival in $\mathrm{N}+$ patients was 11 months ( $\mathrm{SE}=2.828 ; 95 \% \mathrm{Cl}=5.456-16.544$ ) compared to 32 months (SE 6.574, CI 19.116 - 44.884) in the N0 group (Figure 2). For those without lymph node involvement, the 5 -year survival was $31.8 \%$, for those $\mathrm{N}+$, there were no survivors at 5 years. With regards to local control, the $\mathrm{N}+$ median local control was 11 months $(\mathrm{SE}=4.472 ; 95 \% \mathrm{Cl}=$ 2.235 - 19.765) versus an N0 median local control of 28 months ( $\mathrm{SE}=7.041 ; 95 \% \mathrm{Cl}=14.199-41.801)$. Disease-free 5 -year survival was $27.1 \%(\mathrm{~N} 0)$ versus $0 \%(\mathrm{~N}+)$. Post-operative radiotherapy did not confer a survival advantage, either overall or diseasefree, nor did it improve local control. Following post-operative radiotherapy the median survival was 24 months (SE 6.753, $\mathrm{Cl} 10.765-37.235)(n=51)$ whereas with no post-operative radiotherapy, the median survival was 28 months (SE 10.496, Cl 7.428 - 48.572) ( $n=58)$. Median local control with post-operative radiotherapy was 23 months (SE $=2.371,95 \% \mathrm{Cl} 18.353$ - 27.647) versus 21 months ( $\mathrm{SE}=7.287,95 \% \mathrm{Cl} 6.717$ - 35.283) without radiotherapy (Figures 3, 4; Table 1). 
Survival Function

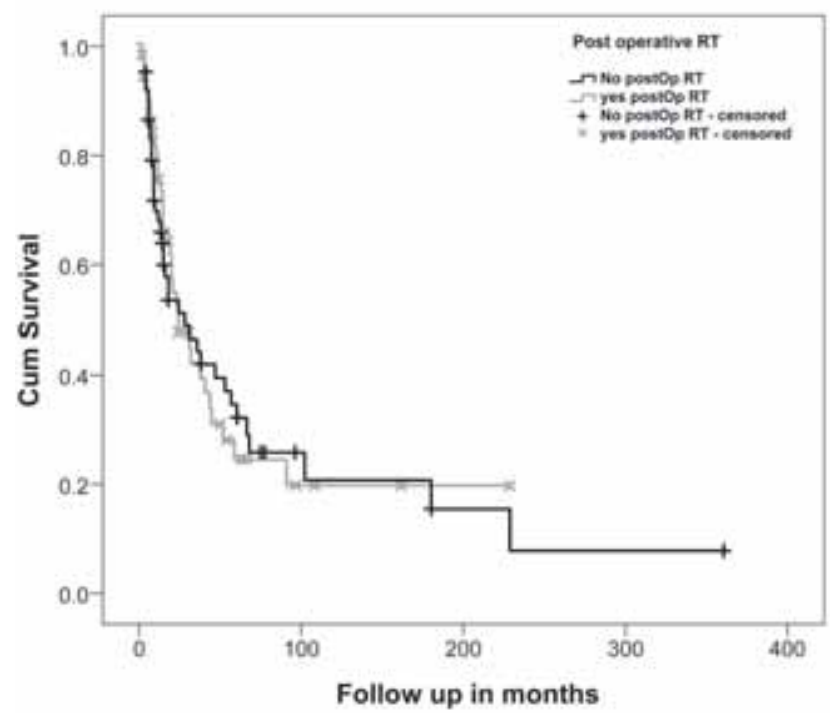

Figure 3. Kaplan - Meier graph of overall survival comparing post-operative radiotherapy to no post-operative radiotherapy.

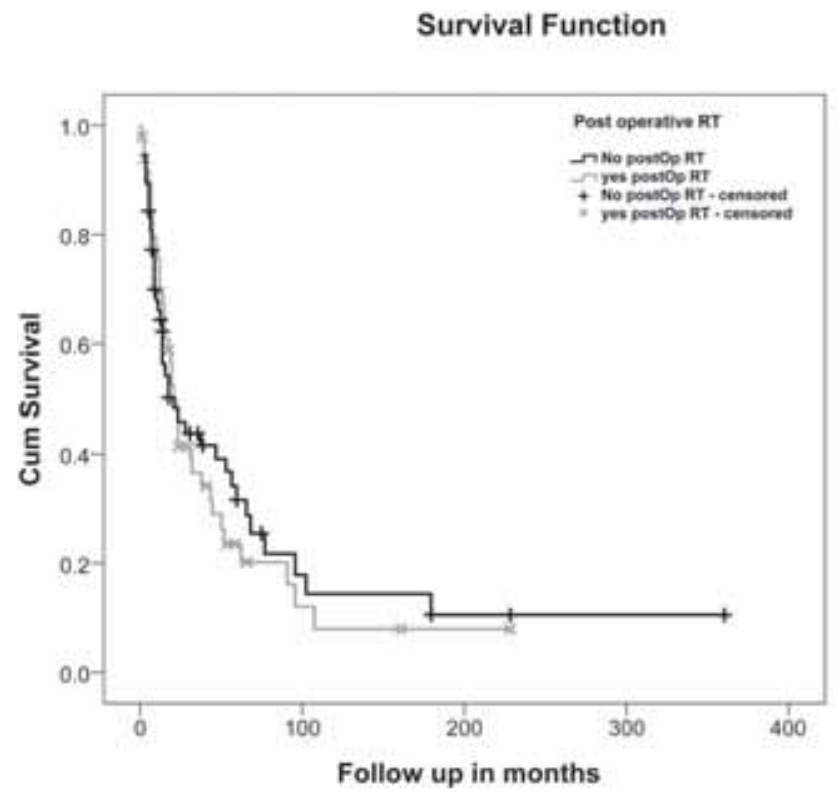

Figure 4. Kaplan - Meier graph of local control of sinonasal melanoma comparing post-operative radiotherapy to no post-operative radiotherapy.
When endoscopic resection was compared to open approach resection there was a significant improved overall survival with endoscopic techniques up to 60 months (Mantel-Cox $p$ $=0.013$ ). There was a similar trend, albeit not reaching statistical significance, favouring the endoscopic technique for local control ( $p=0.225$ ) and disease free survival. Endoscopically resected cases $(n=31)$ had a median 59 month overall survival ( $\mathrm{SE}=17.897 ; \mathrm{Cl} 23.922$ - 94.078) compared to 18 months (SE $=2.228 ; \mathrm{Cl}=13.632-22.638$ ) for open approaches (Figure 5). Open approach resection had a median 18-month local control $(\mathrm{SE}=2.280 ; \mathrm{Cl}=13.532-22.468)$ whereas endoscopic resected cases had a median 50-month local control ( $\mathrm{SE}=18.505 ; \mathrm{Cl}=$ 13.731 - 86.269) (Figure 6). Median disease free survival for endoscopic cases was 36 months $(\mathrm{SE}=17.871 ; \mathrm{Cl}=0.972-71.028$ ) versus 18 months for lateral rhinotomy and midfacial degloving versus 7 months for craniofacial resection and maxillectomy. Five year disease-free survival for these respective groups was $31.3 \%, 22.7 \%$ and $9.1 \%$ ( $p<0.012$ Breslow).

When the surgical technique used was divided into craniofacial resection or maxillectomy $(n=11)$, versus lateral rhinotomy, midfacial degloving or rhinectomy $(n=67)$ versus endoscopic resection $(n=31)$ there was a statistically significant difference between each of the 3 groups' overall survival. Endoscopic surgery carried a better prognosis than the rhinotomy, midfacial degloving or rhinectomy group (median 19 months, $\mathrm{SE}=4.297$; $\mathrm{Cl}=10.578-27.422$ ), which in turn had a better prognosis than the craniofacial resection or maxillectomy group (median 7 month overall survival, $\mathrm{SE}=7.115 ; \mathrm{Cl}=0$ - 20.946) (Figure 7). This was also seen with regard to local control and disease free survival up to 5 years but disappeared thereafter.

Cox regression analysis showed no effect on recurrence, overall survival nor disease free survival for sex, age, site nor for the use of post-operative radiotherapy. Only the presence of lymph nodes conferred a worse outcome.

\section{Discussion}

This is the largest reported series of sinonasal mucosal malignant melanomas from a single institution. The information has been collected prospectively and inevitably covers a period in which both surgical and radiotherapy techniques have changed. Nonetheless, survival is comparable in this study to other reported series which quote a median overall survival of $12.5-19.3$ months or 5 year overall survival of $8 \%$ to $48 \%{ }^{(1,4,6,7,11-}$ 25).

Cutaneous melanomas are staged according to thickness (Breslow), thickness and ulceration (TNM), or in relation to dermal levels (Clarke's levels) ${ }^{(3,8,26,27)}$. These staging systems are backed up by large numbers of cases and closely relate to prognosis. In our series of sinonasal mucosal melanomas, tumours were not staged as a robust system has yet to be demonstrated for mucosal lesions. Sinonasal mucosal melanomas do not lend 
themselves to these staging systems as there are no dermal landmarks, lack of orientation makes measuring depth of penetration difficult and thickness does not strongly relate to outcome. Many staging systems have been proposed for sinonasal melanomas including a new section for mucosal melanomas in the $7^{\text {th }}$ edition of the American Joint Committee on Cancer (AJCC) staging manual (28).

The AJCC omit T1 and T2, making the lowest tumour categorization of mucosal melanoma T3, due to its aggressive behaviour. This leaves a choice of only stage III or IV disease. However, most series have failed to show any relationship between the nasal cavity or sinus TNM staging system for malignant sinonasal melanoma outcomes, the exception being from the M.D. Anderson, TX, USA ${ }^{(9)}$. A simple and frequently used system was proposed by Ballantyne: stage I for localised lesions, stage II for cervical lymph node metastasis and stage III for distant metastasis ${ }^{(29)}$. This has been criticised as most patients present with an array of local disease (stage I) which are not differentiated in this system ${ }^{(24)}$. Prasad et al., proposed a staging system based upon depth of mucosal invasion with Level I defined as melanoma in situ or with 'microinvasion', level II as melanoma invading up to the lamina propria and level III as melanoma with deep tissue invasion ${ }^{(30)}$. They showed this to be an independent predictor of survival. Thompson et al., reviewed 115 cases and proposed a TNM-like system for sinonasal and nasopharyngeal mucosal malignant melanomas where $\mathrm{T} 1$ $=1$ subsite and $\mathrm{T} 2=2$ or more subsites; nodal status was $\mathrm{N} 0$ or
$\mathrm{N} 1$ and the presence of metastatic deposits M1 ${ }^{(1)}$. However, this has not been widely adopted.

In common with some other series, we showed no correlation between tumour site and prognosis ${ }^{(6)}$. Others have suggested that tumours originating in the maxillary or ethmoid sinuses have a worse prognosis than those in the nasal cavity $(7,13,29)$ possibly as a consequence of later presentation or because tumours in these sites are less amenable to resection with clear margins due to orbital or skull base involvement, presumed to be a negative predictive factor ${ }^{(7,31,32)}$.

Surgical resection remains the mainstay of sinonasal melanoma management ${ }^{(2,4,24,33,34)}$. Ideally clear margins should be obtained ${ }^{(25,35)}$ but the proximity or spread of sinonasal tumours to the skull base, orbit and other vital structures makes this a challenge. To achieve this, surgery has traditionally been via an open approach such as lateral rhinotomy or craniofacial resection with its associated morbidity. However, endoscopic resection provides a more detailed, magnified view of the anatomy and accurate assessment of tumour margins than open techniques. Although piecemeal, it allows as complete a resection as that achievable with open approaches and should not be regarded as a less extensive operation ${ }^{(2)}$. Morbidity is reduced with decreased surgical time, decreased hospital stay, less discomfort and improved cosmetic outcome ${ }^{(2,36)}$. Several series have now shown comparable, if not improved outcomes with endoscopic techniques for malignant sinonasal tumours

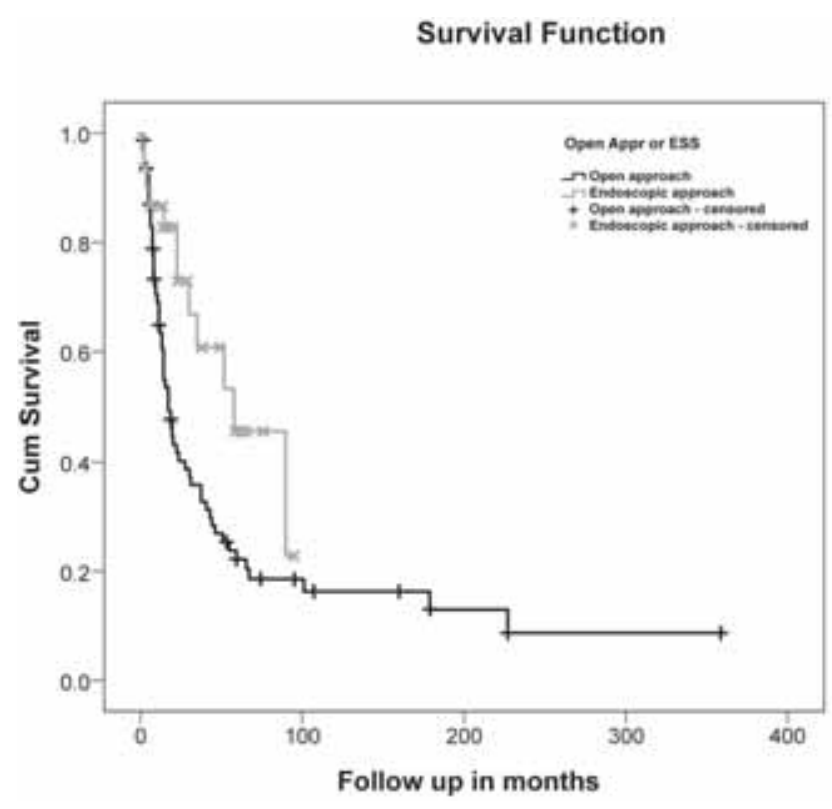

Figure 5. Kaplan - Meier graph of overall survival comparing endoscopic resection (ESS, $n=31$ ) to open approaches $(n=78)$.
Survival Function

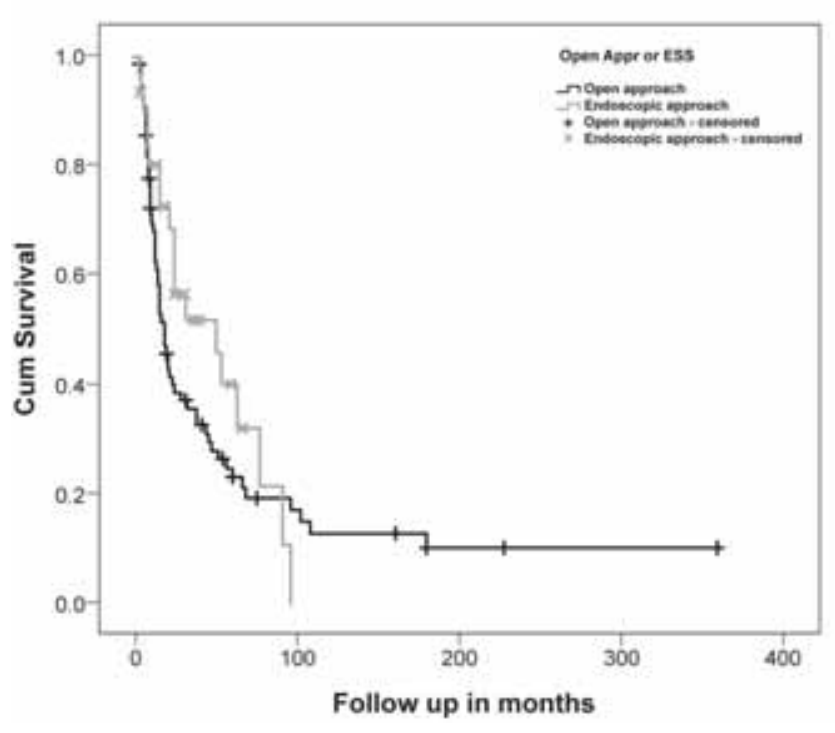

Figure 6. Kaplan - Meier graph of local control comparing endoscopic resection (ESS, $n=31$ ) to open approaches $(n=78)$. 


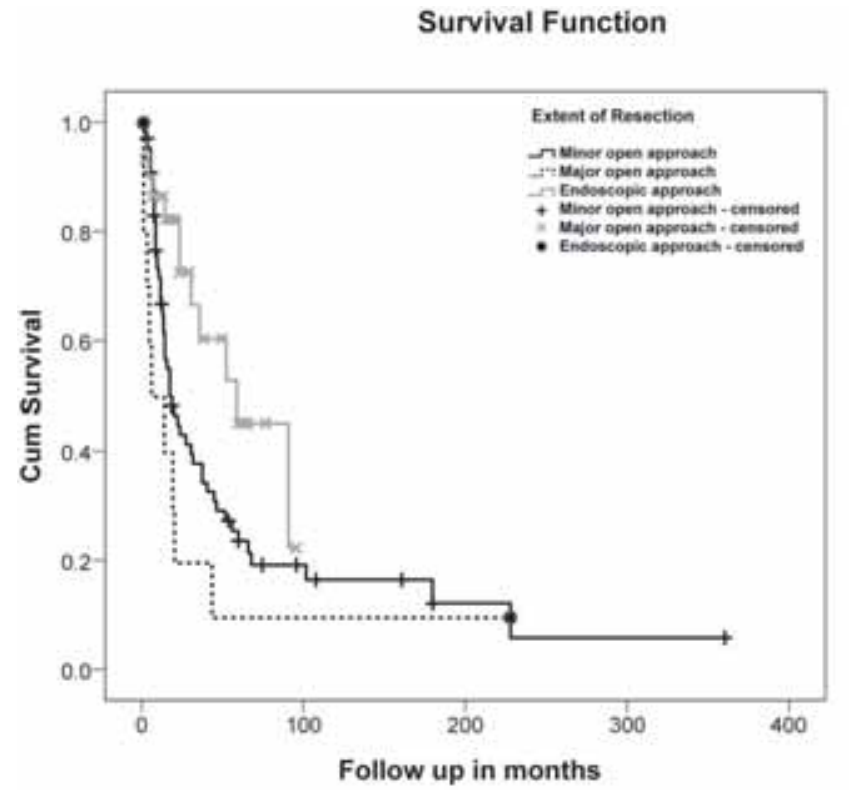

Figure 7. Kaplan - Meier graph of overall survival of 'minor open approach': rhinotomy, midface degloving or rhinectomy $(n=67)$; 'major open approach': craniofacial resection or maxillectomy $(n=11)$ and endoscopic approach $(n=31)$..

when compared to open approaches ${ }^{(7,24,25,32,37)}$. However, it must be emphasised that these are all in selected cases, performed by experienced endoscopic surgeons with curative intent. $A$ possible bias is that in general smaller tumours, associated with a better outcome, are more likely to be selected as candidates for endoscopic removal compared to those with larger, more extensive tumours though there is less evidence that this is of relevance in mucosal malignant melanoma as compared to other sinonasal malignancies. It is also unlikely in this series where endoscopic resection has been undertaken in virtually all patients presenting since the mid-1990's irrespective of extent. At the least, these results indicate that endoscopic resection does not adversely affect the outcome in selected cases with appropriately skilled surgeons and may even improve survival. In sinonasal melanoma this could relate to a reduced disturbance of the exquisitely delicate host-tumour immune balance as a result of the lower morbidity procedure.

In this series radiotherapy was originally administered to cases where complete resection with clear margins had not been achieved or for the more advanced disease. Our first analysis (17), although it did not prove a statistical advantage for radiotherapy, indicated a trend to benefit, which may have been compromised by the numbers of patients available for analysis at that time. Subsequently from 1996, all patients were given the choice of radiotherapy irrespective of extent of disease and this was accepted by 30 of the 50 patients. Whilst this choice
Table 1. Survival statistics, 5 year, 10 year and median for overall survival, disease-free survival and local control.

\begin{tabular}{lccc|}
\hline & $\begin{array}{c}\mathbf{5} \text { year } \\
\text { survival }\end{array}$ & $\begin{array}{c}10 \text { year } \\
\text { survival }\end{array}$ & $\begin{array}{c}\text { Median } \\
\text { (months) }\end{array}$ \\
\hline \multicolumn{1}{|c}{ Overall } & $\mathbf{2 8 \%}$ & $19.4 \%$ & 24 \\
\hline Lymph node & $31.5 \%$ & $21.8 \%$ & 32 \\
N1 & $0 \%$ & $0 \%$ & 11 \\
\hline Radiotherapy & & & \\
None & $31.7 \%$ & $20.3 \%$ & 28 \\
Given & $24.1 \%$ & $19.3 \%$ & 24 \\
\hline $\begin{array}{l}\text { Surgical } \\
\text { approach }\end{array}$ & & & \\
Endoscopic & $45.6 \%$ & $\mathrm{~N} / \mathrm{A}$ & 59 \\
Open Minor & $24 \%$ & $16.9 \%$ & 19 \\
Open Major & $10 \%$ & $10 \%$ & 7
\end{tabular}

\begin{tabular}{lccc}
$\begin{array}{l}\text { Disease-free } \\
\text { Survival }\end{array}$ & $23.7 \%$ & $9.7 \%$ & 21 \\
Lymph Node & $27.1 \%$ & $11.1 \%$ & 24 \\
No & $0 \%$ & $0 \%$ & 11 \\
N1 & & & \\
& & & \\
Radiotherapy & $28.2 \%$ & $12.9 \%$ & 18 \\
None & $18.8 \%$ & $6.5 \%$ & 21 \\
Given & & & \\
& & & \\
Surgical & & & \\
approach & $31.3 \%$ & $11.6 \%$ & 18 \\
Endoscopic & $27.7 \%$ & $0 \%$ & 7 \\
Open Minor & $9.1 \%$ & $11.3 \%$ & 21 \\
Open Major & $\mathbf{2 7 . 7 \%}$ & & \\
\hline Local Control & &
\end{tabular}

Lymph Node

$\begin{array}{cccc}\text { No } & 31.8 \% & 13.0 \% & 28 \\ \text { N1 } & 0 \% & 0 \% & 11\end{array}$

\section{Radiotherapy}

$\begin{array}{llll}\text { None } & 31.9 \% & 14.6 \% & 23 \\ \text { Given } & 23.7 \% & 8.1 \% & 21\end{array}$

Given

$23.7 \%$

$8.1 \%$

Surgical

approach

$\begin{array}{lccc}\text { Endoscopic } & 40.2 \% & \text { N/A } & 50 \\ \text { Open Minor } & 25.3 \% & 12.9 \% & 18 \\ \text { Open Major } & 10.6 \% & 0 \% & 15\end{array}$


contains potential bias, in this larger cohort, if radiotherapy does have some limited benefit, it fails to show statistical significance.

Melanoma is traditionally considered a radio-resistant tumour but may respond to high doses of radiation ${ }^{(38)}$. The few series examining the effects of radiation on mucosal melanoma typically use pooled series from all head and neck sites. These are summarised by Krengli et al., in a recent literature analysis (39). They concluded that post-operative radiotherapy improves local control and recommended its use for unresectable disease but there is no evidence to show it alters survival ${ }^{(39)}$. However, there are several large series, including our own, which fail to replicate this benefit in local control ${ }^{(17,40,41)}$.

With specific regards to the sinonasal cavity, the role of radiotherapy becomes even less clear. The only study to report on radiotherapy as a primary treatment modality resulted in an $18 \% 5$-year survival, lower than most surgical series ${ }^{(38)}$. Moreno et al. 's series from the M.D. Anderson retrospectively looked at 58 cases, 33 of which had received post-operative radiotherapy (9). Although there was no survival benefit, those receiving 54Gy or more had a statistically lower rate of locoregional recurrence than those receiving $30-50 G y{ }^{(9)}$. This further highlights the need for high doses of radiotherapy to achieve any response, which may be limited in sinonasal disease by the close proximity of vital structures such as the optic nerve. Other series, which claim benefit with adjuvant radiotherapy, are based on small numbers and / or lack statistical proof ${ }^{(7,13,38,42)}$. Future developments are likely to include improved staging techniques such as PET-CT, sentinel node assessment, use of molecular markers such as S-100 and tyrosine to identify high risk cases and radiotherapy techniques such as IMRT and neutron beam ${ }^{(39,43,44)}$. The rarity of the tumour will continue to pose the same difficulties in performing randomised prospective trials. Despite the lack of statistical proof, the use of post-operative radiotherapy may be justifiable in patients with sufficient performance status and positive margins after resection or for unresectable disease.

In the literature, the most relevant outcome predictors would seem to be the presence of lymph node involvement (as demonstrated in this series) or distant metastasis ${ }^{(9,24)}$. Locoregional recurrence frequently precedes the occurrence of metastases and may be an independent predictor of survival in sinonasal melanomas ${ }^{(45)}$. Other series have suggested that septal lesions fare better, perhaps because they present earlier or are recognised earlier than their sinus counterparts ${ }^{(13)}$ but conversely there has been a lack of correlation between tumour size and outcome ${ }^{(7)}$ making sinonasal mucosal melanoma one of the most capricious and unpredictable of malignant tumours.

\section{Conclusion}

The prognosis of sinonasal malignant melanoma remains poor with no appreciable overall improvement over recent years. The mainstay of management remains surgical resection. This can be via an open approach such as a lateral rhinotomy but endoscopic techniques may be employed with at least similar outcomes and minimal morbidity. The role of radiotherapy is still unproven and if employed, the optimal radiation doses and fractionation regimes remain undetermined. In an ideal world, a randomized trial could clarify the precise role of radiotherapy and other oncologic options but this will require a large multiinstitutional collaboration in this fortunately rare disease.

\section{Conflict of interests}

None

\section{Authorship contribution}

EC: draft paper, VJL, DJH: surgeons and writing of final paper, WW: statistical analysis.

\section{References}

1. Thompson LD, Wieneke JA, Miettinen M. Sinonasal tract and nasopharyngeal melanomas: a clinicopathologic study of 115 cases with a proposed staging system. Am J Surg Pathol. 2003; 27: 594-611.

2. Lund VJ, Stammberger $\mathrm{H}$, Nicolai $\mathrm{P}$, et al. European position paper on endoscopic management of tumours of the nose, paranasal sinuses and skull base. Rhinology. 2010; 48: 1001-1144.

3. Edge SB, Compton CC, Fritz AG, Greene FL, Trotti A. AJCC cancer staging manual (7th ed). New York: Springer, 2010

4. Mendenhall WM, Amdur RJ, Hinerman RW Werning JW, Villaret DB, Mendenhall NP. Head and neck mucosal melanoma. Am J Clin Oncol. 2005; 28: 626-630.

5. Andersen LJ, Berthelsen A, Hansen HS
Malignant melanoma of the upper respiratory tract and the oral cavity. J Otolaryngol. 1992; 21: 180-185.

6. Bachar G, Loh KS, O'Sullivan B, et al. Mucosal melanomas of the head and neck: experience of the Princess Margaret Hospital. Head Neck. 2008; 30: 1325-1331.

7. Roth TN, Gengler C, Huber GF, Holzmann D. Outcome of sinonasal melanoma: Clinical experience and review of the literature. Head Neck. 2010; 32: 1385-1392.

8. Chang AE, Karnell LH, Menck HR. The National Cancer Data Base report on cutaneous and noncutaneous melanoma: a summary of 84,836 cases from the past decade. The American College of Surgeons Commission on Cancer and the American Cancer Society. Cancer. 1998; 83: 1664-1678.

9. Moreno MA, Roberts DB, Kupferman MEet al. Mucosal melanoma of the nose and paranasal sinuses, a contemporary experience from the $M$. D. Anderson Cancer Center. Cancer; 2010;116:2215-2223.

10. Thompson AC, Morgan DA, Bradley PJ. Malignant melanoma of the nasal cavity and paranasal sinuses. Clin Otolaryngol Allied Sci. 1993; 18: 34-36.

11. Holmstrom M, Lund VJ. Malignant melanomas of the nasal cavity after occupational exposure to formaldehyde. $\mathrm{Br} J$ Ind Med. 1991; 48: 9-11.

12. Huang SF, Liao CT, Kan CR, Chen $\mathrm{H}$. Primary mucosal melanoma of the nasal cavity and paranasal sinuses: 12 years of experience. J Otolaryngol. 2007; 36: 124-129.

13. Dauer EH, Lewis JE, Rohlinger AL, Weaver AL, Olsen KD. Sinonasal melanoma: a clinicopathologic review of 61 cases. Otolaryngol 
Head Neck Surg 2008;138:347-352.

14. Loree TR, Mullins AP, Spellman J, North JH, Jr., Hicks WL, Jr. Head and neck mucosal melanoma: a 32-year review. Ear Nose Throat J. 1999; 78: 372-375.

15. Pandey M, Abraham EK, Mathew A, Ahamed IM. Primary malignant melanoma of the upper aero-digestive tract. Int J Oral Maxillofac Surg. 1999; 28: 45-49.

16. Prasad ML, Busam KJ, Patel SG, HoshawWoodard S, Shah JP, Huvos AG. Clinicopathologic differences in malignant melanoma arising in oral squamous and sinonasal respiratory mucosa of the upper aerodigestive tract. Arch Pathol Lab Med. 2003; 127: 997-1002

17. Lund VJ, Howard DJ, Harding L, Wei WI. Management options and survival in malignant melanoma of the sinonasal mucosa. Laryngoscope. 1999; 109: 208-211.

18. Guzzo M, Grandi C, Licitra L, Podrecca S Cascinelli N, Molinari R. Mucosal malignant melanoma of head and neck: forty-eight cases treated at Istituto Nazionale Tumori of Milan. Eur J Surg Oncol. 1993; 19: 316-319.

19. Stern SJ, Guillamondegui OM. Mucosal melanoma of the head and neck. Head Neck. 1991; 13: 22-27.

20. Narasimhan K, Kucuk O, Lin HS, et al. Sinonasal mucosal melanoma: a 13-year experience at a single institution. Skull Base. 2009; 19: 255-262.

21. Trapp TK, Fu YS, Calcaterra TC. Melanoma of the nasal and paranasal sinus mucosa. Arch Otolaryngol Head Neck Surg. 1987; 113: 1086-1089.

22. Owens JM, Roberts DB, Myers JN. The role of postoperative adjuvant radiation therapy in the treatment of mucosal melanomas of the head and neck region. Arch Otolaryngol Head Neck Surg. 2003; 129: 864-868.

23. Temam S, Mamelle G, Marandas P, et al. Postoperative radiotherapy for primary mucosal melanoma of the head and neck. Cancer. 2005; 103: 313-319.

24. Moreno MA, Hanna EY. Management of mucosal melanomas of the head and neck: did we make any progress? Curr Opin Otolaryngol Head Neck Surg. 2010; 18: 101 106.

25. Clifton N, Harrison L, Bradley P, Jones N.
Malignant melanoma of nasal cavity and paranasal sinuses: report of 24 patients and literature review J Laryngol Otol. 2011; 125: 479-485.

26. Breslow A. Thickness, cross-sectional areas and depth of invasion in the prognosis of cutaneous melanoma. Ann Surg. 1970; 172: 902-908.

27. Clark WH, Jr., From L, Bernardino EA, Mihm MC. The histogenesis and biologic behavior of primary human malignant melanomas of the skin. Cancer Res. 1969; 29: 705-727.

28. Edge SB, Compton CC, Fritz AG, Greene FL, Trotti A. The AJCC Cancer Staging Manual 7th Edition. Springer, 2009.

29. Ballantyne AJ. Malignant melanoma of the skin of the head and neck. An analysis of 405 cases. Am J Surg. 1970; 120: 425-431.

30. Prasad ML, Patel SG, Huvos AG, Shah JP, Busam KJ. Primary mucosal melanoma of the head and neck: a proposal for microstaging localized, Stage I (lymph node-negative) tumors. Cancer. 2004; 100: 1657-1664.

31. McLean N, Tighiouart M, Muller S. Primary mucosal melanoma of the head and neck. Comparison of clinical presentation and histopathologic features of oral and sinonasal melanoma. Oral Oncol. 2008; 44: 1039-1046.

32. Lund VJ, Howard DJ, Wei WI. Endoscopic resection of malignant tumors of the nose and sinuses. Am J Rhinol. 2007; 21: 89-94.

33. Wagner M, Morris CG, Werning JW, Mendenhall WM. Mucosal melanoma of the head and neck. Am J Clin Oncol. 2008; 31 43-48.

34. Howard DJ, Lund VJ, Wei WI. Craniofacial resection for tumors of the nasal cavity and paranasal sinuses: a 25-year experience. Head Neck. 2006; 28: 867-873.

35. Penel N, Mallet $Y$, Mirabel X, Van JT, Lefebvre $J$. Primary mucosal melanoma of head and neck: prognostic value of clear margins. Laryngoscope. 2006; 116: 993-995.

36. Zada G, Kelly DF, Cohan P, Wang C, Swerdloff R. Endonasal transsphenoidal approach for pituitary adenomas and other sellar lesions: an assessment of efficacy, safety, and patient impressions. J Neurosurg. 2003; 98: 350-358.

37. Nicolai $P$, Battaglia P, Bignami $M$, et al. Endoscopic surgery for malignant tumors of the sinonasal tract and adjacent skull base: a 10-year experience. Am J Rhinol. 2008; 22: 308-316.

38. Gilligan D, Slevin NJ. Radical radiotherapy for 28 cases of mucosal melanoma in the nasal cavity and sinuses. Br J Radiol. 1991; 64: 1147-1150.

39. Krengli M, Jereczek-Fossa BA, Kaanders JH, Masini L, Beldi D, Orecchia R. What is the role of radiotherapy in the treatment of mucosal melanoma of the head and neck? Crit Rev Oncol Hematol. 2008; 65: 121-128.

40. Nandapalan V, Roland NJ, Helliwell TR, Williams EM, Hamilton JW, Jones AS. Mucosal melanoma of the head and neck. Clin Otolaryngol Allied Sci. 1998; 23: 107116.

41. Patel SG, Prasad ML, Escrig M, et al. Primary mucosal malignant melanoma of the head and neck. Head Neck. 2002; 24: 247-257.

42. Kingdom TT, Kaplan MJ. Mucosal melanoma of the nasal cavity and paranasal sinuses. Head Neck. 1995; 17: 184-189.

43. Starek I, Koranda P, Benes P. Sentinel lymph node biopsy: A new perspective in head and neck mucosal melanoma? Melanoma Res. 2006; 16: 423-427.

44. Andres R, Mayordomo Jl, Visus C, et al. Prognostic significance and diagnostic value of protein S-100 and tyrosinase in patients with malignant melanoma. Am J Clin Oncol. 2008; 31: 335-339.

45. Lee SP, Shimizu KT, Tran LM, Juillard G, Calcaterra TC. Mucosal melanoma of the head and neck: the impact of local control on survival. Laryngoscope. 1994; 104: 121-126.

\section{Professor V. Lund}

Royal National Throat, Nose and Ear

Hospital

330 Gray's Inn Rd

London

United Kingdom WC1X 8D

E-mail: v.lund@ucl.ac.uk 